Revista Destaques Acadêmicos, Lajeado, v. 8, n. 3, 2016. ISSN 2176-3070 DOI: http://dx.doi.org/10.22410/issn.2176-3070.v8i3a2016.1164 www.univates.br/revistas

\title{
VERIFICAÇÃO DA PRESENÇA DE SALMONELLA SPP EM ALIMENTOS MINIMAMENTE PROCESSADOS EM UM MUNICÍPIO DO INTERIOR DO RIO GRANDE DO SUL
}

\author{
Vanderlea Machado da Silva ${ }^{1}$, Adriana Regina Bitello ${ }^{2}$
}

\begin{abstract}
Resumo: O aumento da incidência de doença transmitida por alimento DTA demonstra que, na atualidade, apesar dos avanços tecnológicos alcançados, este problema ainda ocorre mundialmente. Esse trabalho teve o objetivo de verificar se os alimentos minimamente processados provenientes de um supermercado de um município do interior do Rio Grande do Sul estavam livres de contaminação por salmonela spp. Para isso, foram feitas análises microbiológicas em dois vegetais, que mostraram conformidade com a legislação, ausência de Salmonela spp em 25 gramas de cada amostra.
\end{abstract}

Palavras-chave: Controle de Qualidade. Vegetal Minimamente Processado. Salmonella spp.

\section{INTRODUÇÃO}

Cada vez mais, a segurança dos alimentos é tida como alvo de discussão em muitos estudos, sendo relevante tanto para o consumidor, quanto para fornecedor e fabricante a oferta de produtos que não causem danos à saúde, principalmente com o estilo de vida atual, que faz com que um crescente número de pessoas busque suas refeições fora de casa.

Diante desse contexto, os alimentos minimamente processados (MP) vêm ao encontro da tendência pela maior praticidade e com uma aceitação cada vez maior em todo o mundo (PINTO, 2007). Os produtos MP são procurados por serem compreendidos como produtos frescos, correspondem a um grupo de alimentos que sofreu poucas alterações, como lavagem e sanitização, descascamento, fatiamento, e conservam suas características nutricionais sem adição de produtos químicos (SANTOS; OLIVEIRA, 2012).

\footnotetext{
1 Nutricionista. Aluna do Curso de Pós-Graduação em Gestão da Qualidade e Segurança Alimentar.
}

2 Nutricionista. Professora orientadora. 
Diversas técnicas vêm sendo utilizadas para garantir a disponibilidade de alimentos seguros. No caso de alimentos MP, a manutenção da temperatura de refrigeração durante o processamento e armazenamento é indispensável para manter a qualidade (SANTOS; OLIVEIRA, 2012).

Na década de noventa, o processamento mínimo de frutas e hortaliças iniciou-se no Brasil, encontrando-se em expansão de mercado e de tipos de alimentos disponibilizados ao consumidor nesta forma. O emprego de refrigeração, o aquecimento moderado, embalagem e atmosfera modificada são maneiras de conservar alimentos MP. Estes produtos mantêm as características de um produto fresco, não são estéreis, têm apenas sua carga microbiana reduzida durante o processamento, portanto necessita de cuidados com o armazenamento para impedir o crescimento de microrganismos patogênicos (PIZARRO et al. 2006; ARAUJO et al. 2005; ALVARENGA et al. 2014).

Damasceno et al. (2005) mencionaram ainda que para uma maior segurança seria preciso descriminar no rótulo a exata temperatura que $o$ produto tem que ser armazenado, pois constataram uma variação entre vinte e quatro horas a cinco dias no período de validade de produtos MP, conforme a temperatura de exposição. Embora os vários métodos de conservação se apresentem como uma forma de garantir a qualidade dos alimentos MP, algumas pesquisas têm apontado falhas nesse processo, as quais fazem com que o consumidor seja exposto a riscos de contaminação. Ao encontro disso, vários trabalhos foram realizados com alimentos MP, sendo a Salmonella spp um micro-organismo alvo de análise em muitos deles (DAMASCENO et al., 2005; ÁGUILA et al., 2006; ROMANICHEN et al., 2010).

A ingestão de um alimento contaminado pode ocasionar uma doença transmitida por alimentos, a salmonelose, que é uma das mais comuns e que é causada pela bactéria Salmonella spp, um micro-organismo amplamente distribuído na natureza, sendo o homem e animais de diversas espécies, principalmente as aves são seus principais reservatórios naturais (TRESSELER et al., 2009). Essa patologia pode causar desde sintomas mais brandos até o óbito, dependendo do sorotipo envolvido (SHINORARA, 2008).

A salmonelose é uma das doenças transmitidas por alimentos (DTA) mais preocupantes para a saúde pública em todo o mundo, pelas suas características endêmicas e pelo seu controle depender muito da ação humana, visto que o homem pode ser disseminador dessa bactéria (MATOS et al., 2013). Em grande parte, o fato se deve ao descumprimento das Boas Práticas de Fabricação, ocorrendo, por exemplo, a contaminação cruzada (ROMANICHEN et al., 2010). É importante ressaltar, que a contaminação dos alimentos MP pode ocorrer, também, no início da cadeia, ainda na fase de cultivo, por meio de irrigação com água contaminada ou uso de adubos orgânicos inapropriados (ALVES; NETO; ROSSIGNOLIS, 2013). Por conta disso, se torna indispensável que haja um controle na aquisição da matéria prima, pois, do contrário, o produto final terá grande chance de não ser seguro (ÁGUILA et al., 2006). 
O processamento mínimo aumenta a perecibilidade do produto devido basicamente aos danos mecânicos sofridos durante o processo, tornando a qualidade microbiológica destes produtos assunto de alta prioridade (PINTO, 2007).

Para Martins et al. (2013) é fundamental o uso de métodos adequados para manter a qualidade destes produtos até o consumo final. Em seu estudo, abordam a atmosfera modificada como maneira de preservação, mas ressaltam que muito depende da qualidade inicial do produto, do quão importante são as boas práticas durante todas as etapas do processo, principalmente observar a temperatura baixa que é preciso existir no armazenamento, logística e também na exposição.

Sabe-se que o processo de corte e descascamento nos alimentos MP, liberam algumas enzimas que aceleram a degradação, a polifenol oxidase é um exemplo, ela causa escurecimento, a lipoxidade que no caso de haver, degrada os lipídeos causando cheiro desagradável. O processo de maturação também é acelerado pela respiração que aumenta significativamente nestes processos, por estas razões, a manutenção da qualidade dos alimentos depende do conhecimento destes fenômenos, para que se tenha ações sobre eles a fim de retardar seus efeitos (RAGAERT et al., 2004; ARAUJO; MACHADO; CHITARRA, 2009).

O aumento da incidência de DTA demonstra que, na atualidade, apesar dos avanços tecnológicos alcançados, este problema ainda ocorre mundialmente. Esse trabalho teve o objetivo de verificar a se os alimentos MP, provenientes de um supermercado de uma cidade do interior do Rio Grande do Sul estavam livres de contaminação por Salmonela spp. Para isso, foram feitas análises microbiológicas em dois vegetais.

\section{METODOLOGIA}

Trata-se de um estudo qualitativo exploratório de amostras de vegetais, adquiridos em um supermercado de um município do interior do Rio Grande do Sul. Foram analisadas duas amostras de vegetais MP (cenoura e couve). Os vegetais estavam em uma bandeja de isopor, envolvida por policloreto de vinila. A embalagem estava íntegra, os produtos dentro do prazo de validade e sob refrigeração apropriada. Os itens foram colocados em uma caixa térmica e levados imediatamente a um laboratório de microbiologia da região para serem analisados.

Fez-se a investigação da presença do microrganismo Salmonella spp, seguindo como parâmetro a RDC 12/2001 da Agência Nacional de Vigilância Sanitária (ANVISA), indicada para este tipo de alimento, no que diz que pra Salmonella spp: ausência em 25 gramas de produto. O método utilizado foi Official Method 2011.03 - Salmonella in a Variety of Food - VIDAS® Salmonella 
(SLM) Easy Salmonella Method / ISSO 6579:2002 (E), Microbiology of food and animal feeding stuffs - Horizontal method for the detection of Salmonella spp.

\section{RESULTADOS E DISCUSSÃO}

Ambos os vegetais analisados mostraram ausência de salmonela spp em 25 gramas dos produtos analisados, estando assim de acordo com a legislação, conforme tabela a seguir:

Tabela 1 - Resultados análise microbiológica

\begin{tabular}{|c|c|c|c|c|c|}
\hline $\begin{array}{c}\text { Ensaio } \\
\text { Credenciamento }\end{array}$ & Resultados & Unidade & $\begin{array}{c}\text { 1Limite de } \\
\text { Tolerância } \\
\qquad(*)\end{array}$ & $\begin{array}{l}{ }^{2} \text { Limite de } \\
\text { detecção } \\
\text { Incerteza de } \\
\text { medição }\end{array}$ & $\begin{array}{l}\text { Período } \\
\text { Ensaio M }\end{array}$ \\
\hline $\begin{array}{l}\text { M } 26 \text { - Pesquisa de } \\
\text { Salmonella em } 25 \\
\text { g de amostra de } \\
\text { cenoura }\end{array}$ & Ausência & $25 \mathrm{~g}$ & - & Ausência & $\begin{array}{c}10 / 07 / 2015 \\
a \\
13 / 07 / 2015\end{array}$ \\
\hline $\begin{array}{l}\text { M } 26 \text { - Pesquisa de } \\
\text { Salmonella em } 25 \\
\text { g de amostra de } \\
\text { couve }\end{array}$ & Ausência & $25 \mathrm{~g}$ & - & Ausência & $\begin{array}{c}10 / 07 / 2015 \\
a \\
13 / 07 / 2015\end{array}$ \\
\hline
\end{tabular}

Assim a amostra coletada indicou bom nível qualidade para ser consumida, no que diz respeito a salmonella spp. Diferente deste trabalho, ao analisar a qualidade higiênica sanitária de vegetais MP processados, comercializados na cidade de Maringá no Paraná, verificou-se a presença de salmonela spp, os autores citaram a existência de contaminação cruzada através dos manipuladores, pois o habitat natural deste microrganismo não são os vegetais (ROMANICHEN et al., 2010). Corroborando com estes, o estudo de Tresseler et al. (2009) mostrou que, durante o processo de preparação e comercialização dos MP que analisaram, houveram falhas no processo de higienização, pois foi observada a presença de salmonella spp antes e ao final do processo, perfazendo um total de $12,7 \%$ das amostras coletas impróprias para o consumo.

Santarém (2012) verificou a presença de salmonela spp em amostras de agrião, embora neste trabalho o vegetal não estivesse na forma MP, ou seja, ainda deveria ser higienizado antes do consumo, há de se achar resultado insatisfatório, pois o vegetal não é habitat natural deste microrganismo, portanto tratava-se de um alimento que foi contaminado possivelmente ainda na horta por dejetos humanos e de animais.

Frittoli et al. (2015) não encontraram Salmonela spp nas 20 amostras analisadas, porém, os autores enfatizam que é necessário um melhor controle de qualidade destes produtos, pois embora não houvessem salmonela outros 
microrganismos foram encontrados em $35 \%$ das amostras, os tornando impróprios para o consumo.

No trabalho de Smanioto et al. (2009) não encontraram salmonela spp nas trinta amostras de vegetais MP que foram utilizadas em seu trabalho, estas eram comercializadas na região de Bauru, São Paulo. Apesar disso, também encontraram outros microrganismos patógenos que apontaram deficiência no controle higiênico sanitário.

Alves et al. 2014 encontraram salmonela spp em 2 das 9 amostras analisadas, estas foram adquiridas na rede de supermercados na cidade do Rio de Janeiro. Já em Campinas e região foram analisadas 180 amostras de $\mathrm{MP}$, nenhuma delas mostrou presença de salmonela spp, porém $37,2 \%$ destas estavam impróprias para consumo por terem mostrado outros microrganismos patógenos indicando má qualidade higiênico sanitária (SANTOS et al., 2010).

Um trabalho um pouco diferente também avaliou a qualidade higiênico sanitária de MP em diferentes etapas da cadeia, na saída do processamento mínimo, na unidade produtora de refeição e no balcão de exposição pronto ao consumo, das 35 amostras todas apresentaram ausência de salmonela spp, porém a deficiência no controle higiênico sanitário ficou clara devido as altas contagens de coliformes termotolerantes (KNYCHALA et al., 2010).

Esses resultados comprovam que grande parte do número de contaminação dos alimentos pode ocorrer durante o seu processamento e exige alto cuidado para que sua qualidade e seguridade seja preservada. Embora este estudo apresente algumas limitações, pela baixa representatividade do número amostral e também pela análise restrita de um único microrganismo, no caso só da samonella spp, os resultados são importantes para apontar e discutir a importância do cuidado com o cultivo e manipulação desses vegetais para a saúde da população em geral. Como visto, a samonella spp vem sendo estudada junto com outros microrganismos patógenos em diversas pesquisas que tratam da qualidade higiênico sanitária de alimentos MP e os resultados têm sido preocupantes em muitos casos.

\section{CONSIDERAÇÕES FINAIS}

O estudo da qualidade dos alimentos se torna indispensável para auxiliar na busca da garantia de aumentar a disponibilidade de alimentos seguros para o consumo, assim, ao passo que se analisa um alimento que será comercializado e consumido pela população em geral, se colabora também com o cuidado preventivo na saúde pública.

Apesar de resultados favoráveis e ausência de contaminação por salmonela spp nas amostras em questão, nosso estudo teve como fator limitante o número baixo de amostras analisadas. Nesse sentido, as realizações de mais estudos semelhantes, com número amostral mais representativo poderiam ilustrar melhor o nível da qualidade higiênico sanitária dos produtos 
comercializados nesta região, os quais poderiam corroborar ou confrontar os estudos já realizados em outras regiões do Brasil.

\section{REFERÊNCIAS}

AGUILA, Juan S. Del; SASAKI, Fabiana F.; HEIFFIG, Lilia S.; ONGARELLI, Maria da Graças; GALLO, Cláudio R.; JACOMINO, Angelo Pedro; KLUGE, Ricardo Alfredo. Determinação da Microflora Em Rabanetes Minimamente Processados. Horticultura Brasileira, 24:75-78. Piracicaba, SP, 2006.

ALVARENGA, André Luis Bonnet; TOLEDO, José Carlos; PAULILLO, Luis Fernando de Oriani. Qualidade e Segurança de Vegetais Minimamente Processados: Proposta de estrutura de governança Entre os Agentes da Cadeia e os Sinais de Qualidade. Gestão e Produção. Vol. 21, n. 2, p 341-354, São Carlos, 2014.

ALVES, Thaís Campos; OLIVEIRA, Gabriella Araujo Rebouças; SILVEIRA, Thaís Souza; COSTA Leonardo Emanuel de Oliveira; NASCIMENTO, Janaína dos Santos; OLIVEIRA, Barbara Cristina Euzebio Pereira Dias de; Avaliação da qualidade de vegetais minimamente processados no estabelecimento comercial para vendas a granel. Blucher Food Science Proceedings Novembro de 2014.

ALVES, Ailla da Silva; NETO,Adelino da Cunha; ROSSIGNOLIZ, Paulo Afonso. Parasitos em Alface-crespa (lactuca sativa 1.),de Plantio convencional, Comercializada Em Supermercados de Cuiabá, Mato Grosso, Brasil. Revista de Patologia Tropical, Cuiabá, MG, 2013.

ARAUJO, Francisca Marta Machado Casado de; MACHADO, Antônio Vitor; CHITARRA, Adimilson Bosco. Efeito da Atmosfera Modificada Ativa na Qualidade do Melão. Ciência e Agrotecnologia. Volume 29, 4. 817-823. Lavras, 2005.

BRASIL, Agência Nacional de Vigilância Sanitária, RDC 12, Janeiro de 2001.

DAMASCENO, Karla Suzanne Florentino da Silva Chaves; ALVES, Marta Assunção; MENDONÇA, Silvana Correia de; GUERRA, Nonete Barbosa; STAMFORD, Tânia Lúcia Montenegro. Melão Minimamente Processado: Um controle de qualidade. Revista e Tecnologia Alimentar, 25(4):651-658. Campinas, SP, 2005.

FRITTOLI, Renan Bazuco; RODRIGUES, Luiz Henrique. Análise de coliformes termotolerantes e salmonela sp. em amostras de hortaliças minimamente processadas. Revista Científica da FHO I UNIARARAS. São Paulo. v. 3, 01/2015

MARTINS, Ana Paula Bertoletto, LEVY, Renata Bertazzi; CLARO, Rafael Moreira; MOUBARAC, Jean Claude; MONTEIRO, Carlos Augusto. Participação Crescente de Produtos Ultra Processados na Dieta Brasileira (1987-2009). Revista de Saúde Pública 47: 656-665. São Paulo, SP, 2013.

MATTOS, Leonara M.; MORETTI, Celso Luís; CHITARRA, Adimilson B.; PRADO, Mônica E. T. Qualidade de Alface Crespa Minimamente Processada Armazenada Sob Refrigeração Em Dois Sistemas de Embalagem. Horticultura Brasileira 25: 504508, Lavras, MG, 2007. 
PINTO, Ana Rita da Costa. Qualidade Microbiológica de Frutas e Hortaliças

Minimamente Processadas. Universidade de Brasília. Brasília, 2007.

PIZARRO, Cíntia Alejandra Castilho; BENEDITTI, Benedito Carlos; HAJ-ISA, Niurka Maritza. Avaliação do melão minimamente processado armazenado em diferentes embalagens. Revista Ciência e Tecnologia Alimentar, 26 (2): 246-252. Campinas, SP, 2006.

RAGAERT, P.; VERBEKE, W.; DEVLIEGHERE, F.; DEBEVERE, J. Consumer perception and choice of minimally processed vegetables and packaged fruits. Food Quality and Preference, Essex, v. 15, n. 3, p. 259-270, 2004.

ROMANICHEN, Cristiane; ZIROLDO, Débora Fernanda; SANTOS, Rogério Aparecido Minini dos; SOUZA, Louremi Bianchi Gualda de. Avaliação higiênico sanitária de alimentos minimamente processados. V Mostra Interna de Trabalhos de Iniciação Científica CESUMAR. Maringá, PR, 2010.

PILON, Lucimeire; OETTERER, Marília; GALLO, Cláudio R.; SPOTO, Marta H. F..Schelf life of minimally processed carrot and green pepper. Revista Ciência e Tecnologia Alimentar, 26(1): 150-158. Campinas, SP. 2006.

SANTARÉM, Vamilton Alvares; GIUFFRIDA,Rogério; FABRIS, Paula Andreia. Contaminação de hortaliças por endoparasitas e salmonela spp em Presidente Prudente, São Paulo, Brasil. Chesine Colloquium Agrariae, v. 8, n.1 Jan-Jun. 2012, p. 18-25. DOI: $10.5747 /$ ca.2012. v. 08.n1.a075

SANTOS, Thaís Belo Anacleto dos; JUNQUEIRA, SILVA, Neusely da; Valéria Cristina Amstalden; Microrganismos indicadores em frutas e hortaliças minimamente processadas. Braziliam Journal of Food Technology, Campinas, v. 13, n. 2, p. 141-146, abr./jun. 2010 .

SANTOS, Joana Silva; OLIVEIRA, Maria Beatriz Prior Pinto. Revisão: Alimentos Frescos Minimamente Processados Embalados e em Atmosfera Modificada. Braziliam Journal Food Technology. Campinas, vol.15, p.1-14.2012.

SMANIOTO, Taline Fernanda; PIROLO, Natália Jordão; SIMIONATO, Eliane Maria Ravasi Stéfano; ARRUDA, Maria Cecília de. Qualidade microbiológica de frutas e hortaliças minimamente processados. Rev Inst Adolfo Lutz, Bauru, SP, 2009

TRESSELER, Josiane Fernanda Metler; FIGUEIREDO, Evania Altina Teixeira de; FIGUEIREDO, Raimundo Wilane; MACHADO, Terezinha Feitosa; DELFINO, Camila Martins; SOUZA, Paulo Henrique Machado de. Avaliação da Qualidade Microbiológica de Hortaliças Minimamente Processadas. Ciência Agrotecnologia, v. 33, 1722 -1727, Lavras, 2009.

KNYCHALA, Marília Marques; MELO, Roberta Torres de; MENDONÇA, Eliane Pereira; COELHO, Letícia Ríspoli; Daise Aparecida Rossi. Qualidade microbiológica de vegetais minimamente processados. Publicações em medicina veterinária e zootecnia. Vol. 4 No 35, p 944-950. Maringá, PR, 2010. 